\title{
Live-attenuated Salmonella enterica serotype Choleraesuis vaccine with regulated delayed fur mutation confer protection against Streptococcus suis in mice
}

Yu-an Li $i^{1,2,3,4}$, Yunyun Chen 1,2,3,4, Yuan zhao Dư ${ }^{5}$, Weiwei Guo ${ }^{5}$, Dianfeng Chu' ${ }^{5}$ Juan Fan ${ }^{6}$, Xiaobo Wang ${ }^{1,2,3,4}$, Matthew Bellefleur ${ }^{7}$, Shifeng Wang ${ }^{7}$ and Huoying Shi ${ }^{1,2,3,4^{*}}$ (D)

\begin{abstract}
Background: Recombinant Salmonella enterica serotype Choleraesuis (S. Choleraesuis) vaccine vector could be used to deliver heterologous antigens to prevent and control pig diseases. We have previously shown that a liveattenuated S. Choleraesuis vaccine candidate strain rSC0011 ( $\Delta \mathrm{P}_{\text {crp527:: }}$ TT araC $\mathrm{P}_{\mathrm{BAD}}$ crp $\Delta$ pmi-2426 $\Delta$ relA199::araC $\mathrm{P}_{\mathrm{BAD}}$ lacl $\Pi \Delta$ asdA33, $\Delta$, deletion, $\Pi$, terminator) delivering SaoA, a conserved surface protein in most of $S$. suis serotypes, provided excellent protection against $S$. suis challenge, but occasionally lead to morbidity (enteritidis) in vaccinated mice (approximately 1 in every 10 mice). Thus, alternated attenuation method was sought to reduce the reactogenicity of strain rSC0011. Herein, we described another recombinant attenuated $S$. Choleraesuis vector, rSC0012 ( $\Delta \mathrm{P}_{\text {fur88:: }} \Pi \pi$ araC $\mathrm{P}_{\mathrm{BAD}}$ fur $\Delta p m i-2426 \Delta$ relA199:: $\operatorname{araC} \mathrm{P}_{\mathrm{BAD}}$ lacl $\Pi \mathrm{T}$ asdA33) with regulated delayed fur mutation to avoid inducing disease symptoms while exhibiting a high degree of immunogenicity.

Results: The strain rSC0012 strain with the $\Delta \mathrm{P}_{\text {furs8: }}: T$ araC $\mathrm{P}_{\mathrm{BAD}}$ fur mutation induced less production of inflammatory cytokines than strain rSC0011 with the $\Delta \mathrm{P}_{\text {Crp527:: }}$ TT araC $\mathrm{P}_{\mathrm{BAD}}$ Crp mutation in mice. When delivering the same pS-SaOA plasmid, the intraperitoneal LD $\mathrm{L}_{50}$ of rSC0012 was 18.2 times higher than that of rSC0011 in 3-week-old BALB/C mice. rSC0012 with either pS-SaoA or pYA3493 was cleared from spleen and liver tissues 7 days earlier than rSC0011 with same vectors after oral inoculation. The strain rSC0012 synthesizing SaoA induced high titers of anti-SaoA antibodies in both systemic (IgG in serum) and mucosal (IgA in vaginal washes) sites, as well as increased level of IL-4, the facilitator of Th2-type T cell immune response in mice. The recombinant vaccine rSC0012(pS-SaoA) conferred high percentage of protection against $\mathrm{S}$. suis or $\mathrm{S}$. Choleraesuis challenge in BALB/C mice.

(Continued on next page)
\end{abstract}

\footnotetext{
*Correspondence: hyshi@yzu.edu.cn

'College of Veterinary Medicine, Yangzhou University, Yangzhou 225009, Jiangsu, People's Republic of China

2Jiangsu Co-innovation Center for the Prevention and Control of Important Animal Infectious Diseases and Zoonoses, Yangzhou 225009, China

Full list of author information is available at the end of the article
}

(c) The Author(s). 2020 Open Access This article is licensed under a Creative Commons Attribution 4.0 International License, which permits use, sharing, adaptation, distribution and reproduction in any medium or format, as long as you give appropriate credit to the original author(s) and the source, provide a link to the Creative Commons licence, and indicate if changes were made. The images or other third party material in this article are included in the article's Creative Commons licence, unless indicated otherwise in a credit line to the material. If material is not included in the article's Creative Commons licence and your intended use is not permitted by statutory regulation or exceeds the permitted use, you will need to obtain permission directly from the copyright holder. To view a copy of this licence, visit http://creativecommons.org/licenses/by/4.0/. The Creative Commons Public Domain Dedication waiver (http://creativecommons.org/publicdomain/zero/1.0/) applies to the data made available in this article, unless otherwise stated in a credit line to the data. 
(Continued from previous page)

Conclusions: The live-attenuated Salmonella enterica serotype Choleraesuis vaccine rSC0012(pS-SaoA) with regulated delayed fur mutation provides a foundation for the development of a safe and effective vaccine against $S$. Choleraesuis and S. suis.

Keywords: Salmonella Choleraesuis, Virulence, Immunogenicity, Fur, Inflammatory

\section{Background}

Streptococcus suis is a pandemic pathogen responsible for a wide range of invasive diseases such as pneumonia, meningitis and bacteraemia in both humans and pigs [1, 2]. $S$. suis type 2 (SS2) is the most frequently and virulent isolated from both humans and pigs among all serotypes reported to date $[1,3]$. The surface-anchored protein (Sao) is a highly conserved membrane-anchored protein and proved to be a immunogenic vaccine candidate [4]. However, Sao formulated with Emulsigen-Plus ${ }^{\oplus}$ provides only partial protection to mice against SS2 infection [3]. In our previous study, a recombinant attenuated Salmonella enterica serotype Choleraesuis vaccine strain rSC0016 carrying $s a o A$ gene, provided full protection to mice against SS2 challenge [5]. From the above, an effective delivery system such as live Salmonella enterica serotype Choleraesuis play a crucial role to the effectiveness of Sao.

The use of intracellular Salmonella enterica as a vehicle to deliver heterologous protective antigens against pathogens is an attractive strategy. Curtiss et al. developed the RDAS (Regulated Delayed Attenuated Strategies), which enable live salmonella vaccine effectively colonize lymphoid tissues during the invasion stage because of its wild-type aggressiveness and then be full attenuated by silencing the virulence factor, while stimulate both strong cellular and humoral immunity in the immunized mice [6]. Several ways were used to implement this strategy (RDAS). One way is the reverse synthesis of lipopolysaccharide $\mathrm{O}$-antigen by pmi mutation [7]. Another way is to replace the upstream regulatory and promoter sequences of virulence genes with a tightly regulated araC $\mathrm{P}_{\mathrm{BAD}}$ activator-promoter [8]. This strategy has been successfully used for $S$. Typhimurium and $S$. Typhi [6-8]. With this strategy, we construct a regulated delayed $S$. Choleraesuis vaccine strain rSC0011

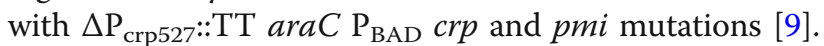
rSC0011 delivering $S$. suis antigens were effective to induce protective immunity against SS2 in mice, but it occasionally caused enteritidis.

We sought to improve our $S$. Choleraesuis candidate vector vaccine by using alternative mutation or introducing new mutation to decreasing its potential to induce enteritidis and enhance immunogenicity. Fur is an important regulatory protein in Salmonella. In the presence of iron, Fur acts as a repressor of iron-controlled genes and mounts an adaptive acid tolerance response
[8]. Synthesis of Fur in a vaccine strain during growth confers acid tolerance and maintains iron homeostasis. A decrease of Fur synthesis in Salmonella leads to acid sensitivity and iron acquisition [9]. Curtiss et al. reported that a $S$. Typhimurium strain with an arabinose regulated delayed fur mutation is highly immunogenic [6]. In these consideration, an arabinose regulated delayed fur mutation $\left(\Delta \mathrm{P}_{\text {fur88: }}: \mathrm{TT}\right.$ araC $\mathrm{P}_{\mathrm{BAD}}$ fur $)$ was introduced into a $S$. Choleraesuis vaccine strain with multiple preexist mutations (Dpmi-2426 $\Delta$ relA199::araC $\mathrm{P}_{\mathrm{BAD}}$ lacI TT $\triangle a s d A 33)$ to generate strain $\mathrm{rSC0012}$. A plasmid pSSaoA [5], encoding saoA from $S S 2$, was transformed into this strain. We evaluated the virulence, immunogenicity and protection against challenge with virulent $S S 2$ or $S$. Choleraesuis C78-3.

\section{Results}

Construction and characterization of the $S$. Choleraesuis vaccine strain rSC0012

Fur is a ferric uptake regulator that is involved not only in iron metabolism, uptake, and transport, but also invasion and survival of $S$. Typhimurium in the hosts [1012]. The absence of Fur attenuates $S$. Typhimurium [6, 13]. To improve the safety and increase the immunogenicity of $S$. Choleraesuis vector, a new strain, rSC0012, was generated with an arabinose regulated fur, $\Delta \mathrm{P}_{\text {fur88:: }}$ TT araC $\mathrm{P}_{\mathrm{BAD}}$ fur (Fig. 1a).

The phenotypes of the mutations $\triangle \mathrm{P}^{\text {fur88}}:: \mathrm{TT}$ araC $\mathrm{P}^{\mathrm{BAD}}$ fur and $\Delta$ relA::araC $\mathrm{P}^{\mathrm{BAD}}$ lacI TT were confirmed by western blot analysis (Fig. 1b). The level of Fur synthesis decreased with arabinose dilution (Fig. 1b). The presence of mutation $\Delta$ relA::araC $\mathrm{P}^{\mathrm{BAD}}$ lacI $\mathrm{TT}$ in rSC0012(pS-SaoA) were confirmed by the increased synthesis of SaoA (Fig. 1b) due to the derepression of $\mathrm{P}_{\text {trc }}$ promoter on plasmid in $\mathrm{rSC} 0012$, which resulted from reduced LacI production whose production was controlled by arabinose. The pmi gene encodes 6phosphomannose isomerase that interconverts fructose6-phosphate and mannose-6-phosphatein in Salmonella [7]. Because mannose is required for O-antigen synthesis, the $\triangle p m i$ mutation enables the strain rSC0012 to display a smooth LPS pattern in nutrient broth in the presence of mannose and a rough pattern in the absence of mannose (Fig. 1c). The $\triangle a s d A$ mutation enables the strain rSC0012 to have an obligate requirement for DAP [14], which can be complemented with a vector 


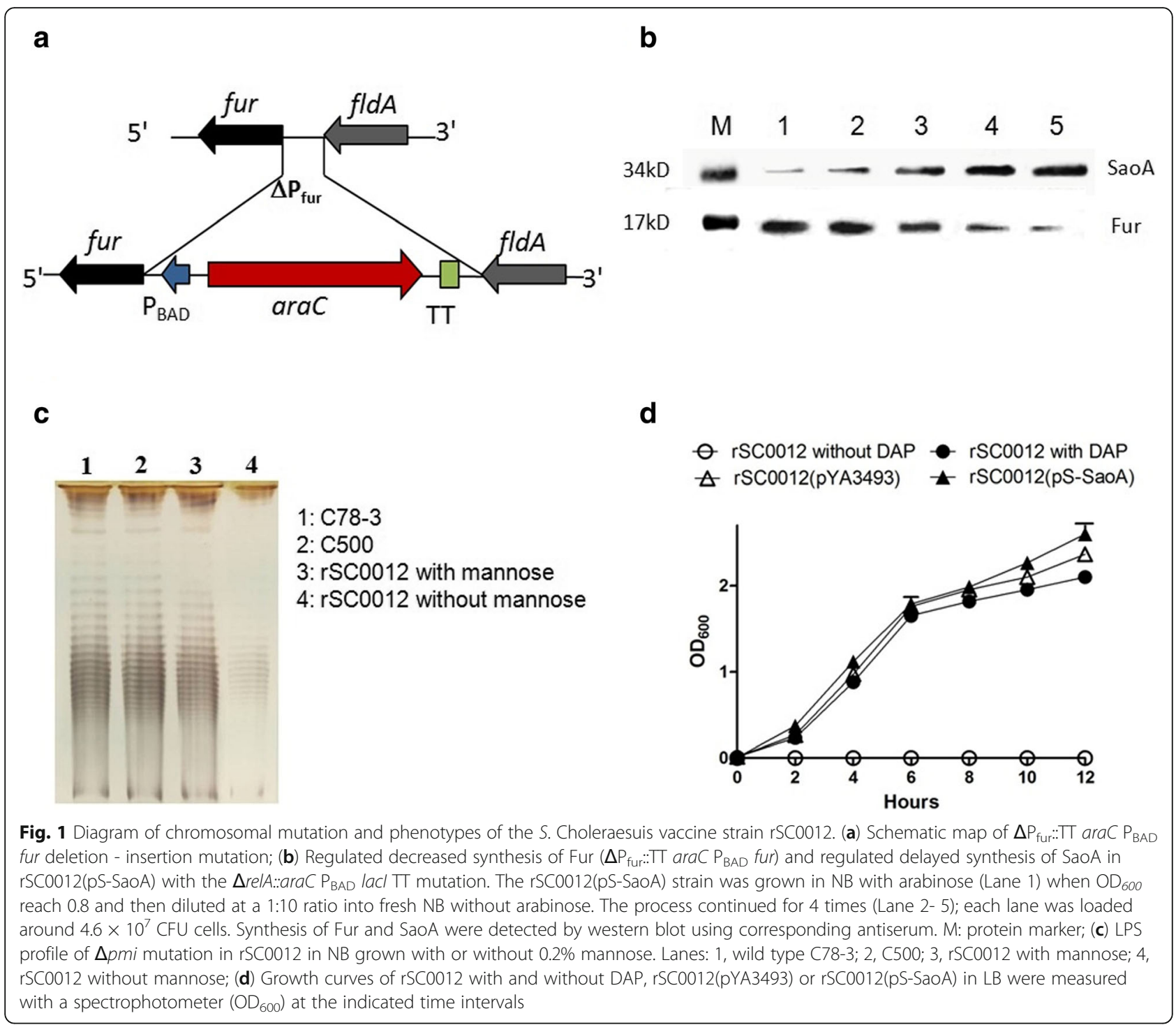

harboring the asd gene then eliminates the need for antibiotic resistance genes for plasmid maintenance [15]. The growth rates of rSC0012 with DAP, rSC0012(pSSaoA), and rSC0012(pYA3493) were similar (Fig. 1d). rSC0012 could grow only with DAP (Fig. 1d).

\section{Antigen synthesis and plasmid stability in S. Choleraesuis rSC0012}

Stable maintenance of plasmids and the production of heterologous antigens are critical to ensure efficacy of recombinant live vaccines. The SaoA protein is a highly conserved surface protective antigen among $S$. suis serotypes $[2,5]$. Using live attenuated $S$. Choleraesuis vector delivering SaoA antigen from S. suis will allow to develop a bivalent vaccine against both $S$. Choleraesuis and $S$. suis. The stabilities of pS-SaoA and pYA3493 in rSC0012 were evaluated by continuous culturing for 50 generations. The stabilities of both $\mathrm{Asd}^{+}$plasmids, pS-SaoA and pYA3493, were $100 \%$ in rSC0012 (data not shown). All rSC0012 colonies examined (100 clones/generation) by endonuclease digestion possessed the Asd + plasmid pS-SaoA or pYA3493. The 34-kDa SaoA protein was detected in cells obtained from both the first and 50th generations of rSC0012(pS-SaoA) (data not shown), indicating the stability of plasmid and stable synthesis of SaoA.

\section{Distribution of secreted SaoA in S. Choleraesuis rSC0012}

The production levels of SaoA in various subcellular fractions from pS-SaoA-carrying strains, rSC0011, rSC0012, and rSC0018, were determined (Fig. 2a-b). rSC0018 is a derivative of C500 [5, 16], a licensed live $S$. Choleraesuis vaccine attenuated by chemical methods in China, with an asdA mutation. The results showed that the level of the SaoA protein produced in the cytoplasm 
a

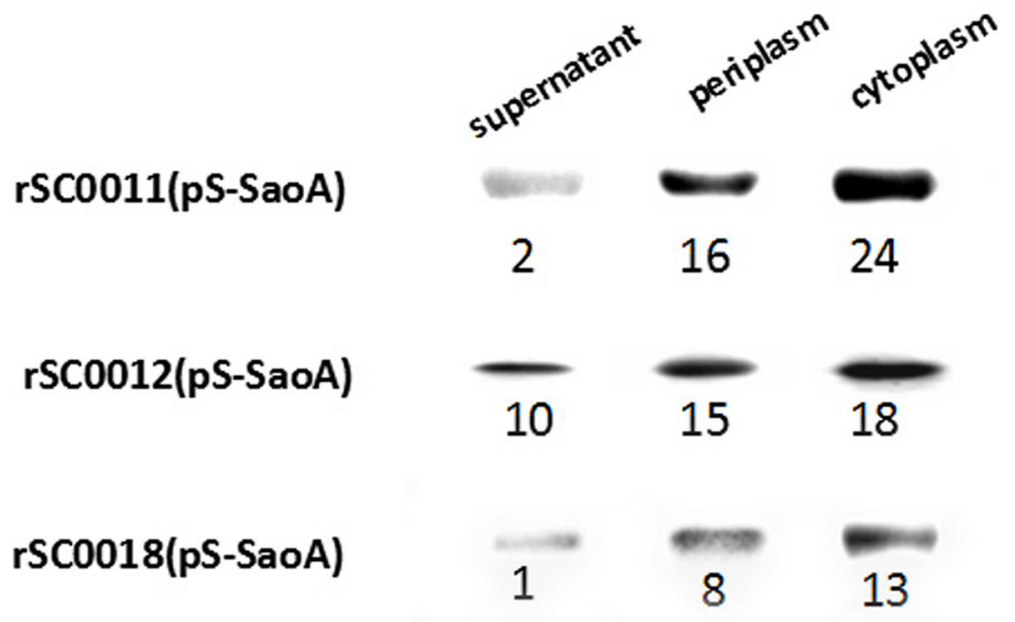

b

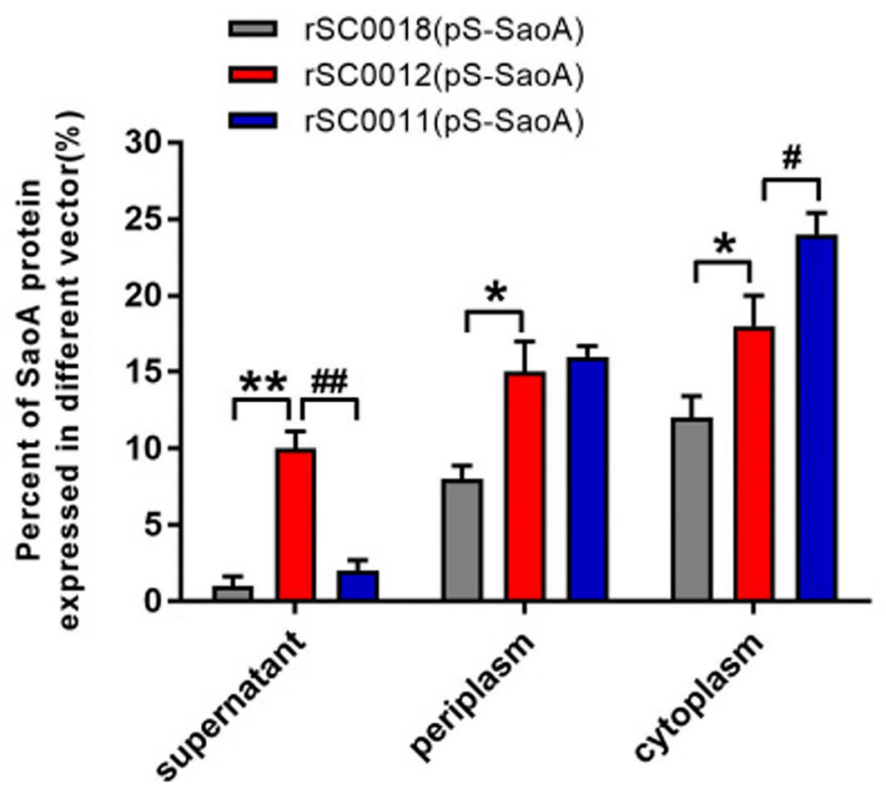

Fig. 2 Synthesis of SaoA in Salmonella Choleraesuis vector rSC0011, rSC0012 and rSC0018. (a) Subcellular fractions of SaoA in rSC0011(pS-SaoA), rSC0012(pS-SaoA), and rSC0018(pS-SaoA) from cells grown in NB detected by western blot. The number showed relative densitometry in one of the three representative experiments. (b) Densitometry analysis of the SaoA protein in rSC0011(pSSaoA), rSC0012(pS-SaoA), and rSC0018(pS-SaoA) by using Image J software (Image J2 PMID 26153368). The assay was repeated 3 times. ${ }^{*} P<0.05,{ }^{* *} P<0.01 . \#, P<0.05, \# \#, P<0.01$, for rSC0012(pS-SaoA) compared to rSC0011(pS-SaoA)

by strain rSC0012(pS-SaoA) was significantly lower than strain rSC0011(pS-SaoA) (Fig. 2b; \#, $P<0.05$ ), but significantly higher in supernatant when compared with rSC0011(pS-SaoA) and rSC0018(pS-SaoA) (Fig. 2b; **, $P<0.01)$. The SaoA protein produced in the periplasm fraction of rSC0012(pS-SaoA) was significantly higher than strain rSC0018(pS-SaoA) (Fig. 2a-b).
Lower virulence of S. Choleraesuis rSC0012 in vivo

To evaluate the virulence of strains with mutations

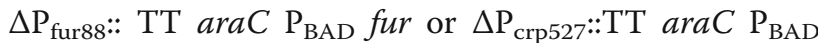
crp, the $\mathrm{LD}_{50}$ values of $\mathrm{rSC} 0012$ and $\mathrm{rSC} 0011$ were tested in 3-week-old BALB/c mice, which represents young mice. rSC0018 was used as an attenuation control. All strains carried the expression plasmid pS-SaoA. 
Table 1 Virulence of rSC0012(pS-SaoA) in 3-week-old BALB/c mice

\begin{tabular}{|c|c|c|c|}
\hline \multirow[t]{2}{*}{ Strain } & \multirow[t]{2}{*}{ Description } & \multicolumn{2}{|l|}{$\mathrm{LD}_{50}(\mathrm{CFU})$} \\
\hline & & Oral & i.p \\
\hline $\mathrm{C} 78-3$ & Wild type & $9.5 \times 10^{2}$ & $<10$ \\
\hline rSC0018(pS-SaoA) & $\triangle$ asdA33 in a live attenuated S. Choleraesuis vaccine strain C500 & $>2.8 \times 10^{9}$ & $6.6 \times 10^{6 * *}, \# \#$ \\
\hline rSC0012(pS-SaoA) & $\Delta \mathrm{P}_{\text {fur88:: }}$ TT araC $\mathrm{P}_{\mathrm{BAD}}$ fur $\Delta p m i-2426 \Delta$ relA199::araC $\mathrm{P}_{\mathrm{BAD}}$ lacl $T \Delta$ asdA33 in C78-3 & $>5.8 \times 10^{9 * *}$ & $2.1 \times 10^{7 * *}, \# \#$ \\
\hline rSC0011(pS-SaoA) & 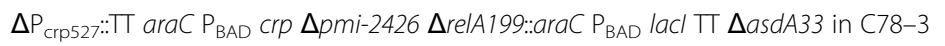 & $>1.0 \times 10^{9 * *}$ & $5.4 \times 10^{5} * *$ \\
\hline
\end{tabular}

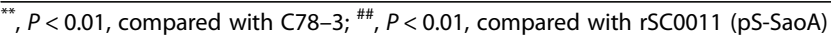

The results revealed that the $\mathrm{LD}_{50} \mathrm{~s}$ of $\mathrm{rSC} 0011(\mathrm{pS}$ SaoA), rSC0012(pS-SaoA), and rSC0018(pS-SaoA) were at least $10^{9} \mathrm{CFU}$ by oral inoculation; whereas, the $\mathrm{LD}_{50}$ of wild-type C78-3 was $9.5 \times 10^{2} \mathrm{CFU}$ (Table 1). Following intraperitoneal infection, the $\mathrm{LD}_{50}$ of $\mathrm{rSC} 0012(\mathrm{pS}$ SaoA) was 38.89-fold higher than that of rSC0011(pSSaoA) and 3.2-fold higher than that of rSC0018(pS$\mathrm{SaoA})$ in mice. These results indicated that the virulence of $\mathrm{rSC} 0012(\mathrm{pS}-\mathrm{SaoA})$ harboring $\Delta \mathrm{P}_{\text {furs8: }}$ : TT $\operatorname{araC} \mathrm{P}_{\mathrm{BAD}}$ fur was significantly lower than that of $\mathrm{rSC} 0011$ (pS-

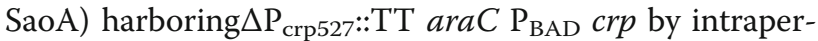
itoneal route in young mice.

\section{Tissue distribution of $S$. Choleraesuis strains in BALB/C mice}

Fur and Crp are important regulatory proteins in Salmonella. Inactivation of the fur and crp genes, attenuates the organism $[6,17]$. To quantitatively the colonization of the $S$. Choleraesuis strains containing regulated delayed fur or crp mutations, 3-week-old BALB/c mice were orally inoculated with rSC0011(pYA3493), rSC0012(pYA3493), rSC0018(pYA3493), rSC0011(pSSaoA), rSC0012(pS-SaoA), rSC0018(pS-SaoA), or wildtype C78-3 (Fig. 3a-f). The mice inoculated with wildtype $S$. Choleraesuis C78-3 died 3-5 days after inoculation, whereas the mice infected orally with vaccine strains rSC0011, rSC0012, rSC0018 containing either plasmid pS-SaoA or pYA3493 survived. The bacteria titers of wild-type strain C78-3 in Peyer's patches, spleen, and liver were significantly higher than those of vaccine strains rSC0011, rSC0012, rSC0018 containing either plasmid pS-SaoA or pYA3493 at 3 days after inoculation (Fig. 3a-c). The titers of bacteria in Peyer's patches were similar for strains rSC0012(pYA3493), rSC0012(pSSaoA), rSC0011(pYA3493), and rSC0011(pS-SaoA) at 321 days post-inoculation (Fig. 3a). At 3d, 7 d, 14 d, 21 d and $28 \mathrm{~d}$, the numbers of $\mathrm{rSC} 0012(\mathrm{pS}-\mathrm{SaoA})$ were significantly higher than those of attenuated vaccine strains rSC0018(pS-SaoA). Same for two control vector, the numbers of rSC0012(pYA3493) were significantly higher than those of attenuated vaccine strains rSC0018(p YA3493) and there was no significant difference with same strain with expression or control vector. Respectively $(P<0.01$; Fig. $3 a)$. These results indicating that the colonization abilities of strains rSC0012(pS-SaoA) and rSC0012(pYA3493) in Peyer's patches were higher than that of rSC0018 with either pYA3493 or pS-SaoA.

In spleen, the titers of rSC0012(pYA3493) and rSC0012(pS-SaoA) were similar to those of vaccine strains rSC0018(pYA3493) and rSC0018(pS-SaoA) at 7, 14, 21, 28 days after inoculation (Fig. 3b). The titers of strains rSC0011(pYA3493) and rSC0011(pS-SaoA) were significantly higher than those of rSC0012(pYA3493), rSC0012(pS-SaoA), rSC0018(pYA3493), and rSC0018(pSSaoA), at 3, 7, 14, and 21 days, respectively (Fig. 3b).

In liver, the titers of rSC0012(pYA3493) and rSC0012(pS-SaoA) were similar to those of vaccine strains rSC0018(pYA3493) and rSC0018(pS-SaoA) at 3, 14, 21, 28 days after inoculation (Fig. 3c). At 3d, 7 d, 14 $\mathrm{d}, 21 \mathrm{~d}$ and $28 \mathrm{~d}$ post-inoculation, the titers of rSC0012(pS-SaoA) were significantly lower than those of rSC0011(pS-SaoA), same for two control vector, the numbers of rSC0012(pYA3493) were significantly lower than those of strains rSC0011(pYA3493).respectively $(P<$ 0.01; Fig. 3c), whereas the strain rSC0012(pYA3493) was the fastest to be cleared in liver (Fig. 3c). These results indicated that the $\Delta \mathrm{P}_{\text {fur88 }}:$ TT araC $\mathrm{P}_{\mathrm{BAD}}$ fur mutation impaired the colonization in the liver of $S$. Choleraesuis vaccine strains. In a summary, the $\Delta \mathrm{P}_{\text {fur88 }}:$ TT $\operatorname{araC}$ $\mathrm{P}_{\mathrm{BAD}}$ fur mutation reduced the colonization ability of $S$. Choleraesuis vaccine strains in mice spleen and liver but not in the Peyer's patches.

\section{Antibody responses in mice immunized with $S$. Choleraesuis strains}

All of the mice immunized with strains containing pS-SaoA developed anti-SaoA antibodies (Fig. 4a). rSC0012(pS-SaoA) induced significantly higher anti-SaoA IgG titer than did rSC0011(pS-SaoA) 3 weeks after the immunization in 3week-old mice (Fig. 4a; *, $P<0.05$ ). Although the anti-SaoA IgG titer of $\mathrm{rSC} 0012(\mathrm{pS}-\mathrm{SaoA})$ were slightly higher than that of rSC0011(pS-SaoA) 5 weeks after immunization, they were not significantly different (Fig. 4a). Compared to mice immunized with rSC0018(pS-SaoA), higher serum IgG titers against SaoA were detected in mice immunized with both rSC0011(pS-SaoA) and rSC0012(pS-SaoA) at 3 weeks and 5 weeks postimmunization (Fig. $4 \mathrm{a}, *, P<0.05$, **, $P<0.01$ ). After boosting, higher titers of anti-SaoA IgG were observed 


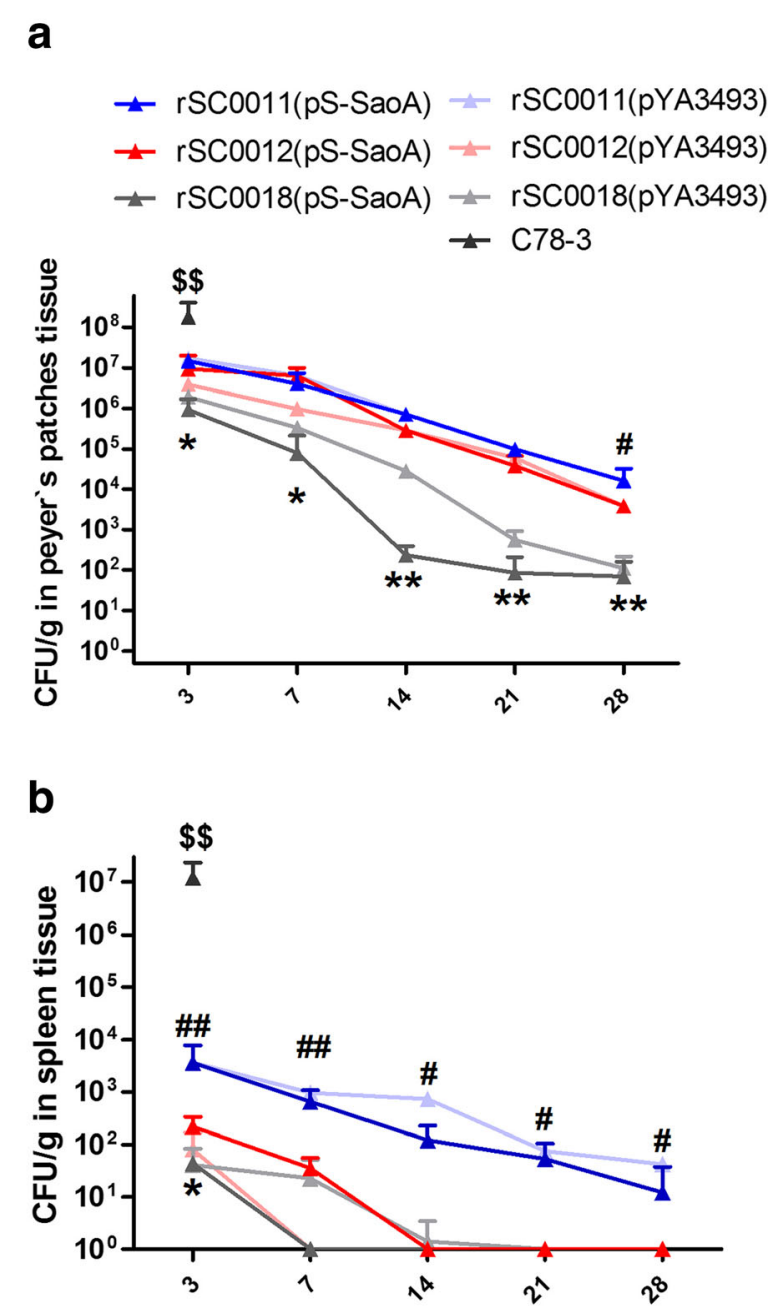

\section{C}

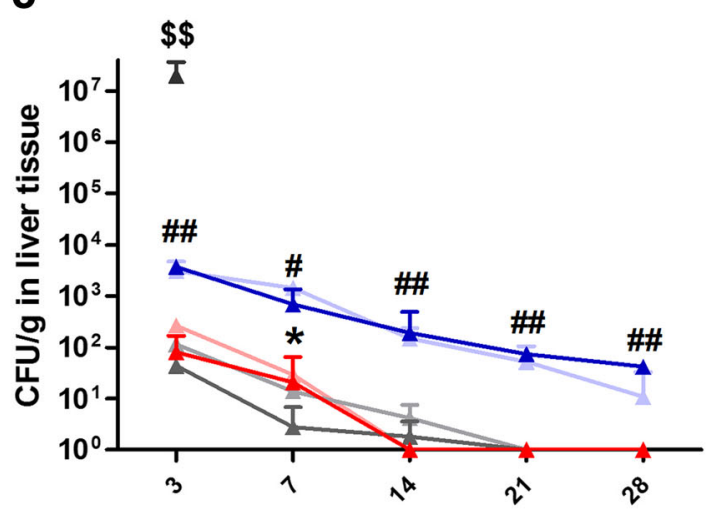

days affter inoculation
Fig. 3 Colonization of Salmonella Choleraesuis rSC0012 in BALB/C mice at diferent time points. Mice were orally inoculated with $1.0 \pm$ $0.3 \times 10^{9} \mathrm{CFU}$ of the indicated strains. The numbers of bacteria loads in the Peyer's patches (a), spleen (b) and liver (c) of mice at 3 $d, 7 d, 14 d, 21 d$ and $28 d$ after inoculation were plotted. Bars represent the arithmetic mean \pm standard deviations from two separate experiments each with 5 mice per group. ${ }^{*}, P<0.05 ;{ }^{*}, P$ $<0.01$, for rSC0018 compared to rSC0012 or to rSC0011 with either PYA3493 or pS-SaoA; \#, $P<0.05$, \#\#, $P<0.01$, for rSC0011 compared to rSC0012 or to rSC0011 with either pYA3493 or pS-SaoA; \$ ,

$P<0.01$, for C78-3 compared to rSC0011, rSC0012 and rSC0018 with either pYA3493 or pS-SaoA, as indicated. The data were collected from two independent experiments

in mice immunized with all three strains containing $\mathrm{pS}-\mathrm{SaoA}$ (Fig. 4a, \#, $P<0.05$ ). All three attenuated recombinant Salmonella strains induced significant OMP titers after the first immunization in mice (Fig. 4b). A significant boosting of serum antibody responses to OMPs was observed after the second immunization, (Fig. 4b; \#, P<0.05).

Mucosal IgA anti-SaoA responses were detected at week 3 in mice immunized with all three attenuated strains containing pS-SaoA. rSC0018(pS-SaoA) induced lower titers of anti-SaoA IgA than rSC0012(pS-SaoA) or rSC0011(pS-SaoA) did in mice (Fig. 4c, *, $\mathrm{P}<0.05$, **, $P<0.01)$. Anti-SaoA IgA levels detected in the rSC0012(pS-SaoA) immunized group were significantly higher than those induced in the rSC0011(pS-SaoA) immunized group at 3 and 5 weeks after the immunization (Fig. 4c, *, $\mathrm{P}<0.05, * *, \mathrm{P}<0.01$ ). These results indicated that strain $\mathrm{rSC} 0012(\mathrm{pS}-\mathrm{SaoA})$ harboring $\Delta \mathrm{P}_{\text {fur88 }}:$ TT araC $\mathrm{P}_{\mathrm{BAD}}$ fur elicited a stronger immune response than

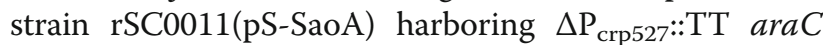
$\mathrm{P}_{\mathrm{BAD}}$ crp did, especially elicit a stronger mucosal immune response.

\section{IFN- $\gamma$ or IL-4 production induced by $S$. Choleraesuis strains}

To further evaluate the effect of the $\Delta \mathrm{P}_{\text {fur88 }}:$ TT araC $\mathrm{P}_{\mathrm{BAD}}$ fur and $\Delta \mathrm{P}_{\text {crp527:: }}$ TT araC $\mathrm{P}_{\mathrm{BAD}}$ crp mutations in a strain with multiple preexist mutations on Th1/Th2 immune responses, the levels of IFN- $\gamma$ and IL-4 in the spleen tissues of mice 7 days and 14 days after booster immunization were measured. The results showed that the titers of IFN- $\gamma$ and IL- 4 induced in mice immunized with rSC0012, and rSC0011 with either pYA3493 or pSSaoA were significantly higher than those induced in mice inoculated by strain rSC0018 with either pYA3493 or pS-SaoA at both 7 and 14 days after booster (Fig. 5a, b; ${ }^{*}, P<0.01$, **, $\left.P<0.05\right)$. Although rSC0011(pS-SaoA) induced a slightly higher level of IFN- $\gamma$ in mice than rSC0012(pS-SaoA) did, no significant difference was observed in 3-week-old mice at both 7 and 14 days after booster (Fig. 5a-c). However, rSC0011(pYA3493) elicited a higher level of IFN- $\gamma$ than rSC0012(pYA3493) did at 7 


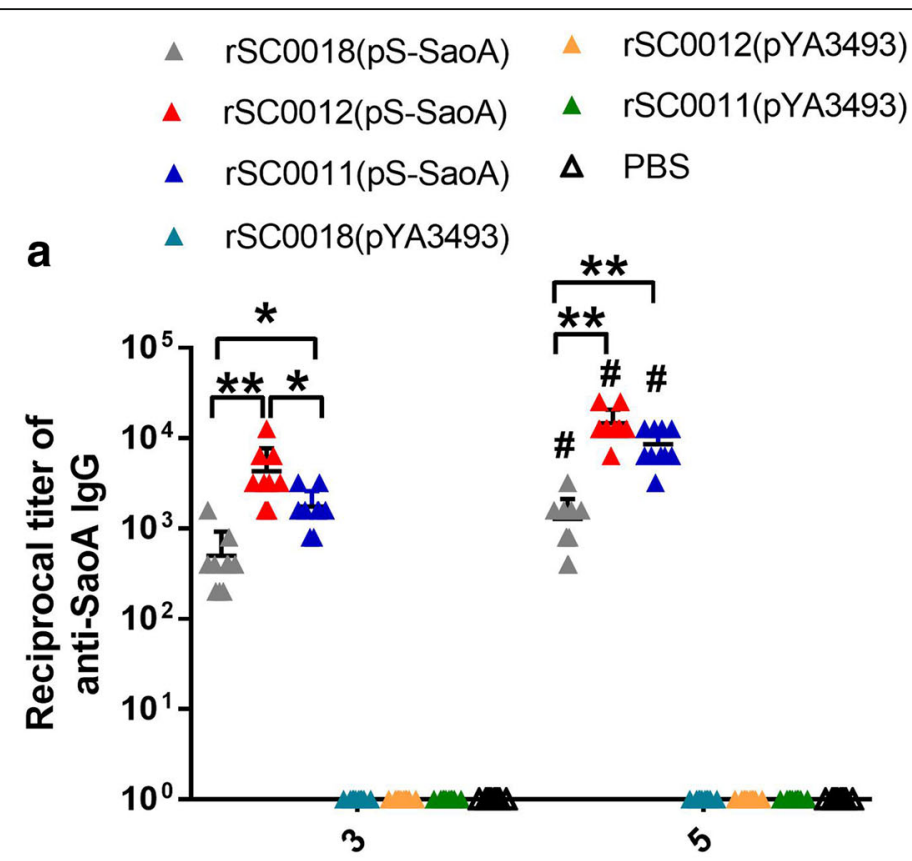

b

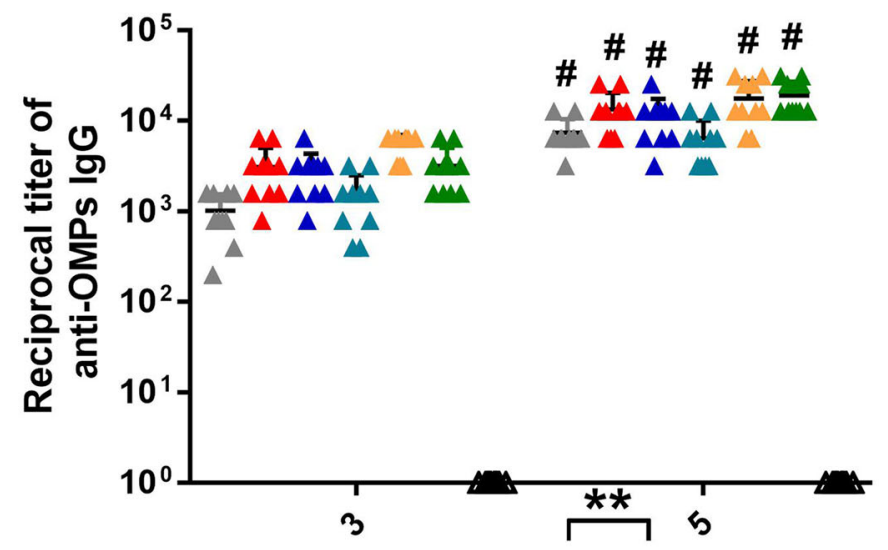

C
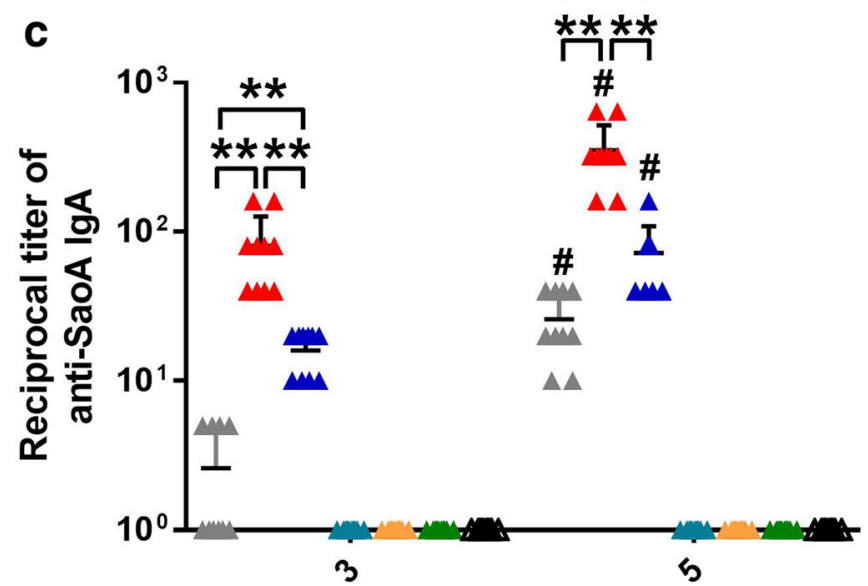

Weeks after immunization 
(See figure on previous page.)

Fig. 4 Antibody responses in ten mice. Serum IgG responses to SaoA (a), S. Choleraesuis OMPs (b) and vaginal wash IgA responses to SaoA (c) were measured by ELISA at weeks 3 and 5. Each triangle represents one mouse. Error bars represent variation between mice. Significant differences were indicated. *, $P<0.05 ;{ }^{* *} P<0.01$, for Salmonella carrying pS-SaoA compared to each other; \#, $P<0.05$; \#\#, $P<0.01$, for the titers of antibody at 3 weeks after immunization were compared to those at 5 weeks after immunization. No immune responses were detected to antigen tested in mice immunized with PBS or in pre-immune sera from vaccinated mice (reciprocal titer <1:50). The assay was performed in duplicate and repeated at least 3 times

days after booster. The titers of IL-4 induced by rSC0012 with either pS-SaoA or pYA3493 in spleen tissues were significantly higher than those induced by rSC0011 with either pS-SaoA or pYA3493 in 3-week-old mice (Fig. 5b; *, $P<0.05, *, P<0.01$ ) at 7 and 14 days after booster. Of note, rSC0012(pS-SaoA) induced a dominant Th2 immune response (IFN- $\gamma<$ IL-4, IFN/IL$4<1$ ) in 3-week-old mice (Fig. 5c); whereas, rSC0011(pS-SaoA) elicited a moderately dominant Th1 immune response (IFN- $\gamma>$ IL-4, IFN/IL-4 $>1$ ) in 3week-old mice (Fig. 5c, ${ }^{*}, P<0.05$, **, $P<0.01$ ). These results indicated the isogenic strain with two different

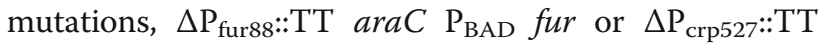
araC $\mathrm{P}_{\mathrm{BAD}}$ crp, affected different branches of immune responses. In additional, the titers of IFN- $\gamma$ and IL-4 induced by the three attenuated strains harboring $\mathrm{pS}-\mathrm{SaoA}$ in immunized mice were higher than those induced by the same strains harboring the emptor vector pYA3493 (Fig. 5a-c; \#, $P<0.05$, \#\#, $P<0.01$ ), suggesting that the protein, SaoA, might augment the immune responses in mice.

\section{Induction of inflammation in mice}

The inflammatory properties of intestinal tissue were investigated in mice after immuned vaccine strains and wild type strain, C78-3.The expression of cytokine genes, TNF $\alpha$, IL- $1 \beta$, IL- 6 and IL- 8 , were assessed by quantitative real -time PCR in gut tissue samples at $6 \mathrm{~h}$ and $12 \mathrm{~h}$ postinfection. All strains with mutation $\triangle \mathrm{P}_{\text {fur88: }}: \mathrm{TT}$ araC $\mathrm{P}_{\mathrm{BAD}}$ fur showed significantly lower transcription levels of cytokine genes IL-1 $\beta$, IL-6, IL-8 and TNF $\alpha$ than the wild -type strain, C78-3 at both $6 \mathrm{~h}$ and $12 \mathrm{~h}$ postinfection (Fig. 6a-d, ${ }^{*}, P<0.05, * *, P<0.01$ ). The C78-3 with mutation $\Delta \mathrm{P}_{\text {fur88: }}: \mathrm{TT}$ araC $\mathrm{P}_{\mathrm{BAD}}$ fur induced significantly lower transcription levels of cytokine genes IL-1 $\beta$, IL- $6, \mathrm{IL}-8$ and TNF $\alpha$ than the same strain with $\Delta \mathrm{P}_{\text {crp527 }}: \mathrm{TT}$ araC $\mathrm{P}_{\mathrm{BAD}}$ crp mutation at both $6 \mathrm{~h}$ and $12 \mathrm{~h}$ postinfection (Fig. $6 \mathrm{c}$ and $\mathrm{d}, \&, P<0.05, \& \&$, $P<0.01)$. At $6 \mathrm{~h}$ postinfection, strain $\mathrm{rSC} 0012(\mathrm{pS}-\mathrm{SaoA})$ showed significantly lower transcription levels of cytokine genes IL-1 $\beta$, IL-6, IL-8 and TNF $\alpha$ than strain rSC0011(pS-SaoA) (Fig. 6a), a similar trend was seen with IL-1 $\beta$, IL-6, IL- 8 and TNF $\alpha$ at $12 \mathrm{~h}$, although the differences were not significant for IL- 6 and TNF $\alpha$ (Fig. 6b,\#, $P<0.05$, \#\#, $P<0.01)$. These results suggest that the addition of the $\Delta \mathrm{P}_{\text {fur8s: }}: \mathrm{TT}$ araC $\mathrm{P}_{\mathrm{BAD}}$ fur mutations could decreased the inflammatory potential of strains rSC0012(pS-SaoA).

\section{Comparison of the protective immunity induced by $S$. Choleraesuis strains}

To evaluate the protective immunity conferred by rSC0012(pS-SaoA), the mice in each immunized group were challenged orally with $50 \times \mathrm{LD}_{50}$ of the virulent $S$. Choleraesuis C78-3 strain or $20 \times \mathrm{LD}_{50}$ of the virulent SS2 strain at 14 days post-boost immunization. After challenge with $\mathrm{C} 78-3$, the results revealed $100 \%$ protection in mice immunized with either strain $\mathrm{rSC0011}(\mathrm{pS}-\mathrm{SaoA})$ or strain rSC0012(pS-SaoA), suggesting full protection. Mice immunized with the rSC0018(pS-SaoA) strains resulted in $20 \%$ survival. In contrast, all the mice in the PBS group succumbed to the challenge after 4 days. There were no significant difference between the groups immunized with rSC0012(pS-SaoA) and rSC0011(pS-SaoA), though both displayed significantly higher levels of protection than the group immunized with rSC0018(pS-SaoA) (Fig. 7a). After the SS2 challenge, immunization with the rSC0011(pSSaoA), rSC0012(pS-SaoA) and rSC0018(pS-SaoA) strains resulted in $80 \%$ survival, $95 \%$ survival and $16.7 \%$ survival with the lethal SS2 challenge, respectively. In contrast, all the mice in the PBS group succumbed to the challenge after 2 days.

\section{Discussion}

The ultimate goal of an engineered live vaccine strain relies on achieving the proper balance between immunogenicity and attenuation [18, 19]. Achieving that goal will restrict unacceptable reactogenicity to avoid overexcitation of inflammatory responses, but sufficient metabolic activity should be maintained to enable the live vaccine to reach deep lymphatic tissues and induce protective immunity [20]. The development of bacterial vaccine relies on a combination of defined mutations $[19,21]$. In order to enhancing the immunogenicity of vaccine strains or to disarm them, multiple independent defined mutations were introduced into Salmonella to generate new recombinant attenuated vaccine strains [6, $19,22]$. By coalescent proper mutations, vaccine strains can be befittingly designed to avoid unacceptable reactogenicity and enhanced immunogenicity [17]. Our previous live attenuated $S$. Choleraesuis vaccine vector rSC0011 occasionally caused enteritidis in mice [9]. One 


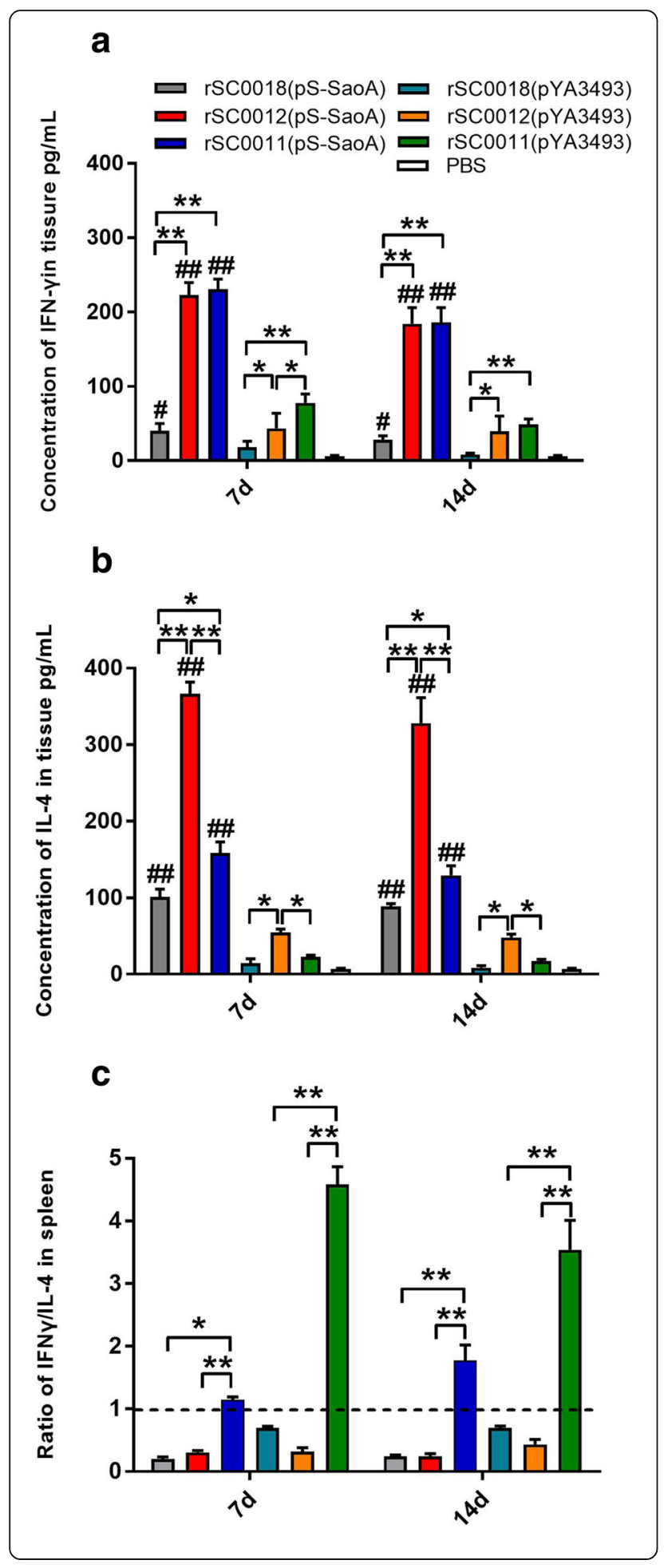

Fig. 5 Cytokines levels in ten mice immunized with the $S$. Choleraesuis vaccines. IFN- $\gamma(\mathbf{a})$ and IL-4 (b) in spleens $7 \mathrm{~d}$ after the booster dose were assayed with an EILSA kit. A PBS control were also included. (c) the ratio of IFN- $\gamma$ to IL-4. The significant differences between groups of each strain was indicated. The assay was performed in duplicate and repeated at least 3 times. ${ }^{*}, P<0.05$; ${ }^{*}$ * $P<0.01$, for strains rSC0011, rSC0012, rSC0018 with either pYA3493 or pS-SaoA compared each other; \#, $P<0.05$; \#\#, $P<0.01$, for the strains rSC0011, rSC0012, rSC0018 with pS-SaoA compared to these strains with pYA3493 respectively at $7 \mathrm{~d}$ and $14 \mathrm{~d}$ post immunization

of the ways to address this problem is by incorporating a sop $B$ mutation $[5,19]$. In this paper, another way to improve the $S$. Choleraesuis vector was reported.

Fur is an important regulatory proteins of Salmonella, which has been implicated in the acid tolerance response since fur mutants are acid sensitive and cause altered expression of several acid shock proteins [10, 23]. A $S$. Typhimurium strain with an arabinose regulated fur mutation is adequately attenuated and highly immunogenic $[6,12]$. However, $S$. Typhimurium belong to group B, while $S$. Choleraesuis group $C$. Whether the $\Delta \mathrm{P}_{\text {furs8: }}: \mathrm{TT}$ araC $\mathrm{P}_{\mathrm{BAD}}$ fur mutation that results in the proper balance between attenuation and immunogenicity of $S$. Typhimurium is also appropriate for attenuated $S$. Choleraesuis vaccine has not been reported previously.

We corroborated the $S$. Choleraesuis strain rSC0012 $\left(\Delta \mathrm{P}_{\text {fur88: }}: \mathrm{TT}\right.$ araC $\mathrm{P}_{\mathrm{BAD}}$ fur $\Delta p m i \Delta$ relA::araC $\mathrm{P}_{\mathrm{BAD}}$ lacI TT $\triangle a s d A$ ) displayed a regulated decrease of Fur production in the absence of arabinose. In a previous publication, $S$. Typhimurium strain with a single $\Delta \mathrm{P}_{\text {furs8: }}:$ TT $\operatorname{araC}$ $\mathrm{P}_{\mathrm{BAD}}$ fur mutation has shown higher virulent than $S$. Typhimurium with a single $\Delta \mathrm{P}_{\text {crp527: }}$ TT araC $\mathrm{P}_{\mathrm{BAD}}$ crp mutation by an oral immunization. Unlike report from Curtiss et al. in $S$. Typhimurium [6], our studies did not showed that the $\Delta \mathrm{P}_{\text {fur88: }}: \mathrm{TT}$ araC $\mathrm{P}_{\mathrm{BAD}}$ fur mutation has

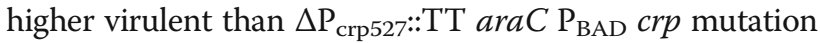
in $S$. Choleraesuis. In fact,the $\mathrm{LD}_{50}$ of rSC0012(pS-SaoA) was 38-fold higher than rSC0011(pS-SaoA) for 3-weeksold mice with intraperitoneal injection. This result suggest that the $\Delta \mathrm{P}_{\text {fur88: }}: \mathrm{TT}$ araC $\mathrm{P}_{\mathrm{BAD}}$ fur mutation does different attenuation with $S$. Choleraesuis or $S$. Typhimurium, which may due to its complex role as a transcriptional activator of virulence in different strains [24].

The $\Delta \mathrm{P}_{\text {fur88: }}: \mathrm{TT}$ araC $\mathrm{P}_{\mathrm{BAD}}$ fur mutation can modify the iron regulated outer membrane protein, then strain with $\Delta \mathrm{P}_{\text {fur88::TT araC }} \mathrm{P}_{\mathrm{BAD}}$ fur induced lower inflammation than the isogenic strain with $\Delta \mathrm{P}_{\text {crp527 }}: \mathrm{TT}$ araC $\mathrm{P}_{\mathrm{BAD}}$ crp mutation or wild type strain in mice. Theses results suggest that the $S$. Choleraesuis with $\Delta \mathrm{P}_{\text {fur88 }}:$ TT $\operatorname{araC} \mathrm{P}_{\mathrm{BAD}}$ fur mutation exhibits a lower tendency to trigger excessive inflammation while attenuated sufficiently.

Through the oral vaccination route, a vaccine strain will endure the challenges of acid, bile, and antimicrobial 


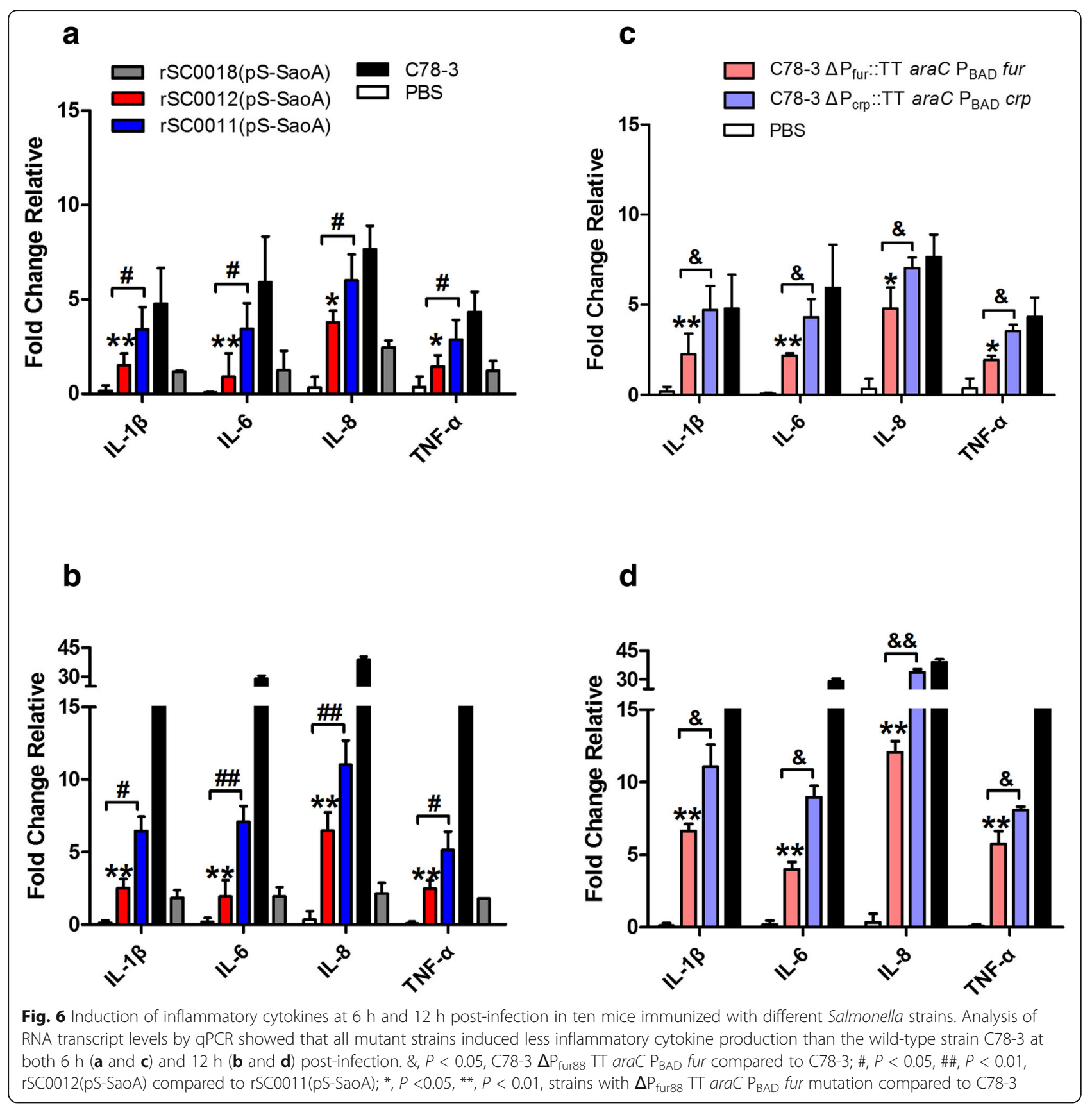

peptides existing in the gastrointestinal tract. Once inside Peyer's patch, it will face macrophagocytes and $\mathrm{T}$ cells during the process transferring to deep lymphatic tissues [13]. Fur is essential to Salmonella for accessorial an adaptive acid tolerance response [10]. Although both rSC0011 and rSC0012 had similar levels of Peyer's patch colonization by oral immunization,we observed that the rSC0012 was cleared more rapidly than the rSC0011 in deeper lymphatic tissues, spleen, and liver of mice. These results suggest that strain rSC0012 was more attenuated than rSC0011 in deep lymphoid tissue, which maybe due to an increase in acid sensitivity cause by loss of Fur makes the cell more susceptible to killing by macrophages.

Both rSC0012 and rSC0011 induced higher levels of IgA, IgG, IFN- $\gamma$, and IL4 responses with great colonization compared to strain rSC0018 with less colonization in mice. In general, the live Salmonella vaccines with RDAS display superior colonization level in lymphoid tissues during the invasion stage, leading to enhanced protection by effectively colonizing lymphoid tissues [6, 25]. However, there are do exist high levels of colonization but low immunogenicity. A strain with the regulated delayed $r f c$ mutation exhibits superior colonization and yet does not 


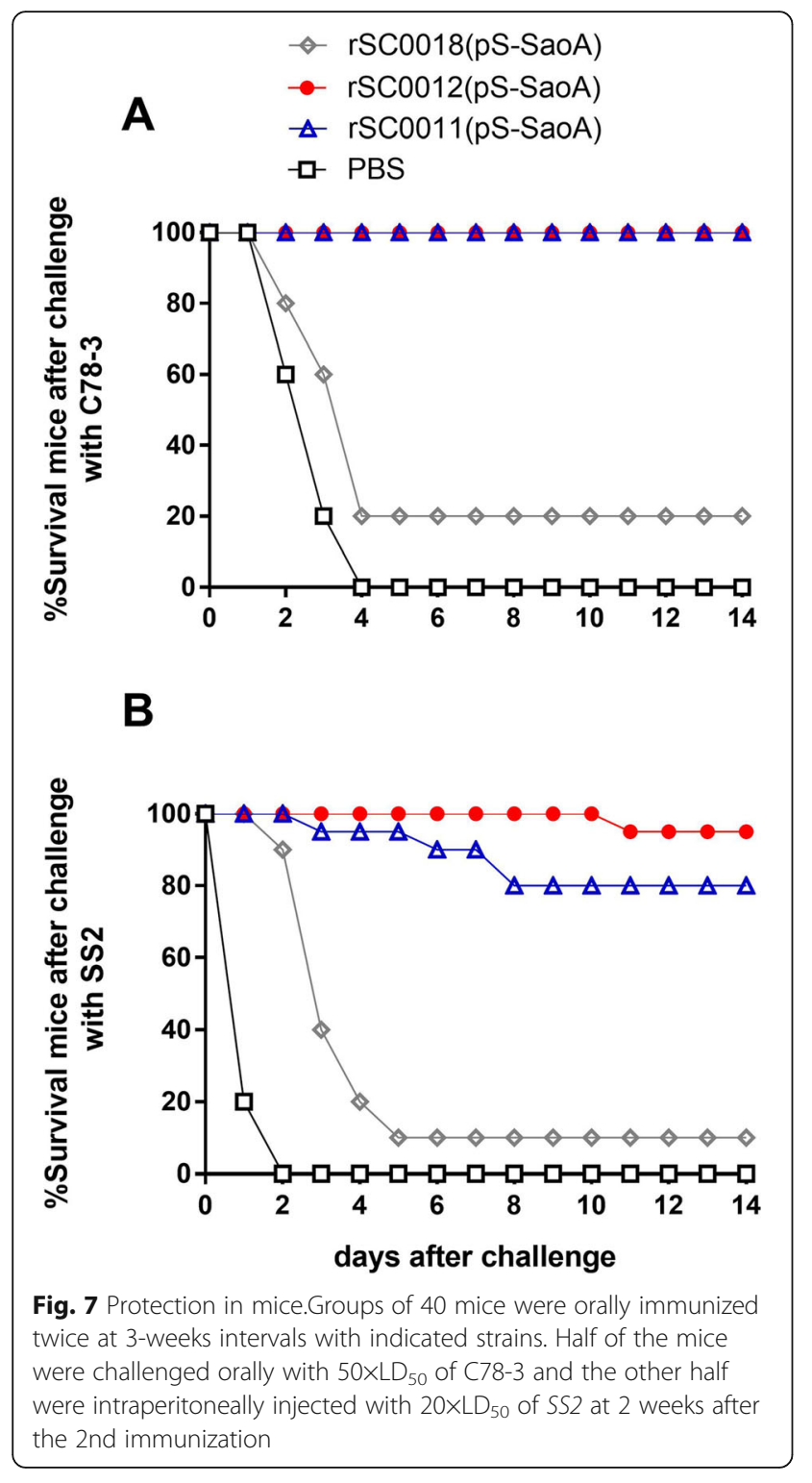

stimulate higher heterologous protection than a $\Delta r f c$ strain without RDAS [26]. Thus, in addition to colonization level, other determining factors may exist to induce the enhanced protection achieved by regulated delayed attenuation. From the above, Selecting the proper mutation is critical for vaccine development with RDAS. This study confirmed above statement. Although the colonization of $\mathrm{rSC} 0012(\mathrm{pS}-\mathrm{SaOA})$ in mice was less than that of rSC0011(pS-SaoA), rSC0012(pS-SaoA) stimulated stronger serum IgG and mucosal IgA responses than the rSC0011(pS-SaoA). This phenomenon suggests that strain with regulated delayed fur mutation may stimulate stronger antibody response with fewer bacteria than strain with regulated delayed crp mutation.

Both rSC0012 and rSC0011 aroused a Th1 cellmediated response, as ostensived by the significant up- regulation of imprint Th1 cytokines, IFN- $\gamma$, the stimulator of Th1-type $\mathrm{T}$ cell immune response. It could partially be that the intracellular characteristics of Salmonella enterica cause them to be detected on the surface of APC through MHC-I molecules. This result is consistent with previous studies with $S$. Typhi [6]. Strain rSC0012 induced higher Th2 cell-mediated response than strain rSC0011, as aroused by the significant up-regulation of imprint Th2 cytokines, IL-4 and greater humoral responses than rSC0011 in mice. This phenomenon may be related to the secreting of heterologous antigen. The more heterologous antigens were secreted during infection, the more likely to trigger an IL-4-dominant response [27]. We found a larger secretion of the SaoA from rSC0012, which could be presented by MHC-II molecules. An additional factor may be due to an increase in acid sensitivity cause by loss of Fur makes the rSC0012 more susceptible to killing by macrophages, thus weakening rSC0012's ability to survive in macrophages. The presence of S. suis-specific IgA serves to promptly deliver the antigen to Peyer's patch dendritic cells or phagocytes and also promptly excite the adaptive immunity during secondary exposure [16]. Generally speaking, the more attenuated the vaccine strain is, the lower its immunogenicity is $[17,22]$, while, the more attenuated rSC0012 strain induced significantly higher IgA and IgG antibody responses to SaoA than the rSC0011 at 3 weeks after the initial immunization in juvenile mice. This may be the result of a equilibrium between attenuation and the ability to participation immune components and activate a controlled over-inflammatory response by the finely crafted strain [19].

The results presented herein highlight that strain rSC0012(pS-SaoA) with regulated delayed fur mutation has retained vaccine efficacy and adequate immunogenicity whilst being safer than previous strain rSC0011(pSSaoA). Furthermore, the inclusion of the $\Delta \mathrm{P}_{\text {furs8 }}:$ TTar$a C P_{\mathrm{BAD}}$ fur mutation may be able to decrease inflammation caused by live-attenuated Salmonella enterica serotype Choleraesuis vaccine, which has been an imperfection for other live-attenuated Salmonella enterica serotype Choleraesuis vaccine which transformed from wild type virulent strain. Both $S$. Choleraesuis and S. suis are major swine pathogens. Currently, there exist license live vaccines against $S$. Choleraesuis for swine, including Entersol ${ }^{\circ}$ Salmonella $\mathrm{T} / \mathrm{C}$ and SC-54 manufactured by Boehringer Ingelheim and Arugs SC/ST, respectively [28]. However, there is no licensed vaccine against $S$. suis. Preventing the diseases caused by SS2 in swine with a combined vaccine is a long-sought goal.

\section{Conclusions}

Our results have shown the strains rSC0012(pS-SaoA) with regulated delayed fur mutation could confer higher protection against challenges with lethal doses of $S S 2$ or 
S. Choleraesuis C78-3. Thus, the use of attenuated $S$. Choleraesuis to develop a vaccine against $S$. suis will have the great potential to ease the burden of both pathogens. The regulated delayed fur mutation in the novel vaccine rSC0012 resulted in a well-safety, highly immunogenic, and effective vaccine in mice, this study has paved the way for testing in piglets. Our findings will aid the optimal of a $S$. Choleraesuis vaccine vector capable of eliciting a suitable immune response against other pathogens.

\section{Methods}

\section{Animals}

Three-week-old female BALB/c mice were purchased from Animal Center of Yangzhou University, and kept 1 week before inoculation. All animal experiments were authorized by the Jiangsu Administrative Committee for Laboratory Animals (permission number SYXK-SU2007-0005) and accorded to the Jiangsu Laboratory Animal Welfare and Ethics guidelines of the Jiangsu Administrative Committee of Laboratory Animals. All surgery was performed under anesthesia intraperitoneally injected with sodium pentobarbital, $40 \mathrm{mg}$ per kilogram mouse weight. All the animals were humanely euthanized after the study by inhalation of $\mathrm{CO}_{2}$, while injection with sodium pentobarbital, $40 \mathrm{mg}$ per kilogram mouse weight, and all efforts were made to minimize suffering.

\section{Strains, plasmids, and culture conditions}

The strains, plasmids, used in this study are described in Table 2. C500, an approved live $S$. Choleraesuis vaccine strain attenuated by thallium compound in China, was used as an attenuation control [32]. The genetic characterization of this strain has been reported [29]. $S$. suis serotype 2 (SS2, CVCC3928) and S. Choleraesuis C78-3 (CVCC79103) were purchased from China Institute of Veterinary Drug Control. Plasmid pYA3493 is an Asd $^{+}$vector with a $\mathrm{P}_{\text {trc }}$ promoter. The asd gene from Salmonella was used as a unique plasmid marker to be used in asd mutants to constitute a balanced-lethal system [14]. LB medium [5],Nutrient broth (NB) and MacConkey agar (Difco) were used for phenotype characterization. When required, media were supplemented with 2,6-diaminopimelic acid (DAP;50 $\mu \mathrm{g} / \mathrm{mL}$ ), chloramphenicol $(\mathrm{Cm} ; 25 \mu \mathrm{g} / \mathrm{mL}), \mathrm{L}$-arabinose $(0.2 \% \mathrm{wt} /$ $\mathrm{vol})$, D-mannose $(0.2 \% \mathrm{wt} / \mathrm{vol})$ or sucrose $(5 \% \mathrm{wt} / \mathrm{vol})$. The empty plasmid vector pYA3493 and expression vector pS-SaoA were described on previous studies [5]. The saoA gene is under the control of the $\mathrm{P}_{\text {trc }}$ promoter (Table 2). S. Choleraesuis vaccine vector strain rSC0012 harboring plasmid pS-SaoA (expression vector) or pYA3493 (control vector) were grown in LB broth with both $0.2 \%$ arabinose and $0.2 \%$ mannose. Selenite broth was used for enrichment of $S$. Choleraesuis from mice tissues. Strains were prepared as previously described [5, $6,9,12]$. Bacterial growth was monitored with a spectrophotometer at $\mathrm{OD}_{600}$ and by direct plating for colony counts.

\section{Construction of $\mathrm{S}$. Choleraesuis mutant strains}

Four mutations $\Delta \mathrm{P}_{\text {fur88 }}: \mathrm{TT}$ araC $\mathrm{P}_{\mathrm{BAD}}$ fur, $\Delta p m i$, $\Delta$ relA::araC $\mathrm{P}_{\mathrm{BAD}}$ lacI $\mathrm{TT}$, and $\triangle a s d A$ were introduced into $S$. Choleraesuis $\mathrm{C} 78-3$ by conjugation with $E$. coli X7213 harboring aforementioned suicide vectors as previously described [33]. The suicide vectors used are listed in Table 2 and Fig. 1a. To construct mutation $\Delta \mathrm{P}_{\text {fur88:: }}$ TT araC $\mathrm{P}_{\mathrm{BAD}}$ fur, a 1335-bp $\mathrm{TT}$ araC $\mathrm{P}_{\mathrm{BAD}}$ cassette were used to replace the 239-bp promoter sequence of the fur gene to achieve arabinose-regulated Fur synthesis (Fig. 1a). The araC $\mathrm{P}_{\mathrm{BAD}}$ cassette contains a transcription terminator (TT) sequence to prevent araC transcription reading through adjoining genes. Plasmid for $\Delta \mathrm{P}_{\text {fur88 }}:$ TT araC $\mathrm{P}_{\mathrm{BAD}}$ fur were confirmed by DNA sequencing. All the primers used have been reported [21].

\section{Characterization of $S$. Choleraesuis mutations in vitro}

All mutations were confirmed by colony PCR using homologous primers [12]. The $\triangle a s d A$ mutation was verified by growth with or without DAP in LB broth [14, 15]. Lipopolysaccharide (LPS) profiles were examined by silver staining in $12 \%$ sodium dodecyl sulfatepolyacrylamide (SDS) gel for the $\Delta p m i$ mutation [33, 34]. The $\Delta \mathrm{P}_{\text {furs8 }}:$ TT araC $\mathrm{P}_{\mathrm{BAD}}$ fur deletion-insertion mutation was verified by reduced production of Fur protein as arabinose concentrations decreased with the increased bacterial growth by western blot using anti-Fur antiserum [12]. The production of SaoA was verified by western blot using anti-SaoA antiserum, respectively $[9$, $18,19,20$,$] .$

\section{Salmonella subcellular fractionation}

To evaluate the subcellular localization of synthesized SaoA in the live attenuated $S$. Choleraesuis vaccine, cultures were grown in $\mathrm{NB}$ to an $\mathrm{OD}_{600}$ of 0.8 and centrifuged at $13,200 \times$ g for $5 \mathrm{~min}$ to collect supernatant and pellet. The culture supernatant was saved for later analysis of the bacterial secreted proteins. Periplasmic fractions were prepared using the lysozyme-osmotic shock method as previously described [27, 35]. Equal volumes of supernatant,periplasmic, cytoplasmic samples were separated by SDS-PAGE. Proteins were then transferred to polyvinylidene fluoride membrane for western blot analysis using anti-SaoA antiserum [5]. The gel band were analyzed with ImageJ software (NIH) [31]. 
Table 2 Strains and plasmids

\begin{tabular}{|c|c|c|}
\hline Strain or plasmid & Relevant characteristics or genotype & Source or reference \\
\hline \multicolumn{3}{|l|}{ E. coli strains } \\
\hline BL21 & $\mathrm{F}^{-} \mathrm{p} T$ hsdSB (rB' $\mathrm{mB}^{-}$al $d c m$ (DE3) & Invitrogen \\
\hline$x 7213$ & $\begin{array}{l}\text { thi-1 thr-1 leuB6 fhuA21 lacY1 glnV44 asdA4 recA1 } \\
\text { RP4 2-Tc::Mu pir; Km }\end{array}$ & Gift from Dr. Curtiss, III \\
\hline \multicolumn{3}{|l|}{ Salmonella Choleraesuis } \\
\hline C78-3 & Wild type, virulent, CVCC79103 & Lab stock \\
\hline C500 & $\begin{array}{l}\text { S. Choleraesuis vaccine strain attenuated by chemical } \\
\text { mutation, CVCC79500 }\end{array}$ & {$[29]$} \\
\hline rSC0005 & $\Delta p m i-2426$, C78-3 & {$[9]$} \\
\hline rSC0008 & $\Delta p m i-2426 \Delta$ relA199::araC $P_{\mathrm{BAD}}$ lacl TT & This study \\
\hline rSC0009 & $\begin{array}{l}\Delta p m i-2426 \text { relA199::araC } \mathrm{P}_{\mathrm{BAD}} \text { lacl } \mathrm{TT} \\
\Delta \mathrm{P}_{\text {fur88::TT }} \text { araC } \mathrm{P}_{\mathrm{BAD}} \text { fur }\end{array}$ & This study \\
\hline rSC0011 & $\begin{array}{l}\Delta \mathrm{P}_{\text {crp527::TT araC }} \mathrm{P}_{\mathrm{BAD}} \text { crp } \Delta p m i-2426 \\
\text { relA199::araC } \mathrm{P}_{\mathrm{BAD}} \text { lacl TT } \Delta \text { asdA33 }\end{array}$ & {$[9]$} \\
\hline rSC0012 & $\begin{array}{l}\Delta p m i-2426 \Delta \text { relA199::araC } \mathrm{P}_{\mathrm{BAD}} \text { lacl } \mathrm{TT} \\
\Delta \mathrm{P}_{\text {fur88: }} \text { TT araC } \mathrm{P}_{\mathrm{BAD}} \text { fur } \Delta \text { asdA33 }\end{array}$ & This study \\
\hline \multirow[t]{3}{*}{ rSC0018 } & $\triangle a s d A 33$ & C500 \\
\hline & C78-3 $\Delta \mathrm{P}_{\text {fur88:: }}$ TT araC $\mathrm{P}_{\mathrm{BAD}}$ fur & C78-3 \\
\hline & C78-3 $\Delta \mathrm{P}_{\text {crp527:: }}$ TT araC $\mathrm{P}_{\mathrm{BAD}} \mathrm{crp}$ & C78-3 \\
\hline Streptococcus suis serotype 2 & Wild type, virulent, CVCC3928 & Lab stock \\
\hline \multicolumn{3}{|l|}{ Plasmids } \\
\hline pYA3493 & $\begin{array}{l}\text { Asd }^{+} ; \mathrm{pBR} \text { ori, } \mathrm{P}_{\mathrm{trc}} \text { promoter, } \beta \text {-lactamase signal } \\
\text { sequence-based periplasmic secretion plasmid }\end{array}$ & {$[15]$} \\
\hline pS-SaoA & pYA3493 with SaoA, $P_{\text {trc }}$ promoter & {$[5]$} \\
\hline pET28a & expression vector, $\mathrm{T} 7$ promoter; $\mathrm{Km}^{\mathrm{r}}$ & Novagen \\
\hline \multicolumn{3}{|l|}{ Suicide vector } \\
\hline pRE112 & SACB mobRP4 R6K oriV oriT $\mathrm{Cm}^{r}$ & {$[30]$} \\
\hline pDMS197 & tet' sacB mobRP4 R6K oriV oriT & {$[30]$} \\
\hline pYA3832 & $\Delta \mathrm{P}_{\text {crp527 }}: \because T$ araC $\mathrm{P}_{\mathrm{BAD}}$ crp, pRE112 & {$[31]$} \\
\hline pYA3546 & $\Delta p m i-2426, \mathrm{pDMS197}$ & {$[31]$} \\
\hline pS003 & DrelA199::araC $\mathrm{P}_{\mathrm{BAD}}$ lacl TT, pRE112 & {$[9]$} \\
\hline pS005 & $\Delta P_{\text {furs8:: }}: T$ araC $P_{B A D}$ fur & This study \\
\hline pYA3736 & $\triangle$ asdA33, pRE112 & {$[9]$} \\
\hline
\end{tabular}

\section{Preparation of SaoA and Salmonella outer membrane proteins (SOMPs)}

His-tagged SaoA fusion protein and Salmonella outer membrane proteins (SOMPs) were prepared as previous studies [5].

\section{Determination of virulence in mice}

Three-week-old female BALB/c mice were obtained from Animal Center of Yangzhou University. Mice were fasting for $6 \mathrm{~h}$ before inoculation. Recombination $S$. Choleraesuis vector strains were cultured in $\mathrm{LB}$ broth with $\mathrm{D}-0.2 \%$ mannose and $0.2 \%$ L-arabinose for $12 \mathrm{~h}$ at $37^{\circ} \mathrm{C}$ as standing cultures. The cultures were diluted 1:50 in the same media and cultured at $37{ }^{\circ} \mathrm{C}$ to an $\mathrm{OD}_{600}$ of 0.9 . Bacteria were collected by centrifugation at $13,200 \times \mathrm{g}$ for $5 \mathrm{~min}$ at room temperature and resuspended in PBS to densities suitable for the inoculation. Serial dilutions of the $S$. Choleraesuis strains were plated onto LB agar supplemented with $0.2 \% \mathrm{D}$-mannose and $0.2 \% \mathrm{~L}$-arabinose to measure the actual densities. Groups of five mice were inoculated with different doses in $20 \mu \mathrm{l}$ (oral immunization) or $100 \mu \mathrm{l}$ (intraperitoneal immunization). The mice were observed for 4 weeks for death. The LD50s were calculated according the method of Reed and Muench [10].

\section{Distribution of Salmonella in BALB/c mice}

A colonization assay for recombination $S$. Choleraesuis vector strains was performed as described previously [9, 10]. Three-week-old female BALB/c mice were divided into 7 groups with 25 mice in each group. Each mouse 
was orally inoculated with $1 \pm 0.2 \times 10^{9} \mathrm{CFU}$ of $S$. Choleraesuis strains. Peyer's patches, spleen, and liver of the mice were collected on days $3,7,14,21$ and 28 postinoculated. The densities of bacteria in the tissues were determined using the method reported in previous studies $[5,9,10,36]$. The assay was performed twice, and the data were similar and pooled for analysis.

\section{Immunization of mice}

Bacteria were grown and collected as above. Serial dilutions of the $S$. Choleraesuis vaccine strains were plated onto LB agar supplemented with $0.2 \% \mathrm{D}$-mannose and $0.2 \% \mathrm{~L}$-arabinose to determine the actual dose. Threeweek-old female BALB/c mice were orally inoculated with $1 \pm 0.2 \times 10^{9} \mathrm{CFU}$ of $S$. Choleraesuis vaccine strains containing either pS-SaoA or pYA3493. Mice were boosted inoculated with the same dose of the same strain after 3 weeks. About $50 \mu \mathrm{L}$ of whole blood was collected by tail vein 3 weeks after primary inoculation and 2 weeks after boosting. Serum was separated from the whole-blood samples and stored at $-70{ }^{\circ} \mathrm{C}$. Vaginalwash samples in mice were collected at the indicated time and stored at $-70^{\circ} \mathrm{C}[9,25,30,36]$. This experiment was performed in triplicate with each group receiving a similar dose of the vaccine strains.

\section{Tissue collection after Salmonella infection of mice}

Three-week-old female BALB/c mice were divided into 5 groups with 10 mice in each group. Groups of mice were orally inoculated with $1 \pm 0.3 \times 10^{9} \mathrm{CFU}$ of Salmonella strains. Spleen and intestinum tenue of the mice were collected at $6 \mathrm{~h}$ and $12 \mathrm{~h}$ postinfection. The tissues were frozen with liquid nitrogen and then transferred to $-70^{\circ} \mathrm{C}$.

\section{Enzyme-linked immunosorbent assay (ELISA)}

Serum IgG antibody production against $S$. Choleraesuis outer membrane proteins (OMPs) and SS2 SaoA,and vaginal-wash IgA antibody production against SaoA in mice were evaluated by Enzyme-linked immunosorbent assay (ELISA) [5]. Cytokines in tissues were analyzed by sandwich ELISA using commercial kits (BD Biosciences) according to manufacturer's instructions. The results from the two experiments were pooled for statistical analysis.

\section{Quantitative real -time PCR (qPCR) for cytokines}

For RNA isolation, gut tissues were homogenized and suspended in TRIzol $^{\circ}$ (Thermo Fisher Scientific,USA). Tubes were vortexed for $3 \mathrm{~min}$ to disrupt the tissues. Chloroform was added to TRIzol ${ }^{\circ}$-treated samples and the samples centrifuged at $13200 \times \mathrm{g}$ for $10 \mathrm{~min}$. The aqueous phase was separated out, and the RNA precipitated using precooled isopropanol. For quantitative realtime PCR, $1 \mu \mathrm{g}$ of RNA was then reverse transcribed to
Table 3 Primer sequences

\begin{tabular}{lll}
\hline Gene & & Sequence $\left(5^{\prime}-3^{\prime}\right)$ \\
\hline GAPDH & Forward & CTT AGC ACC CCT GGC CAA G \\
& Reverse & GAT GTT CTG GAG AGC CCC G \\
IL-1 $\beta$ & Forward & GTG TCT TTC CCG TGG ACC TT \\
& Reverse & AAT GGG AAC GTC ACA CAC CA \\
IL-6 & Forward & GGC GGA TCG GAT GTT GTG AT \\
& Reverse & GGA CCC CAG ACA ATC GGT TG \\
TNF-a & Forward & ATG AGC ACA GAA AGC ATG A \\
& Reverse & AAG AGG CTG AGA CAT AGG C \\
IL-8 & Forward & CTG CAA GAG ACT TCC ATC CAG \\
& Reverse & AGT GGT ATA GAC AGG TCT GTT GG \\
\hline
\end{tabular}

cDNA. The primers were designed using Primer Blast (NCBI net) and synthesized by TSINGKE Biological Technology Co., Ltd. The sequences of the primers are listed in Table 3. Each sample were amplified using 7500 Fast Real -Time PCR Instrument (ABI,US) using Fast SYBR Green Master Mix (Thermo -Fisher Scientific). The results were using internal reference GAPDH as control for normalization, and the $2^{\wedge^{-\Delta \Delta C t}}$ method was used to estimate the relative expression level of the mRNAs of target genes.

\section{Challenge with S. suis serotype 2 (SS2) and $S$. Choleraesuis (C78-3) in mice}

Twenty mice in each group were challenged with $S S 2$ by intraperitoneal (i.p.) injection with $2.4 \times 10^{8} \mathrm{CFU}$ of $S S 2$ in $100 \mu \mathrm{l} \mathrm{PBS}$ at 5 weeks after primary immunization. The $50 \%$ lethal dose $\left(\mathrm{LD}_{50}\right)$ of $S S 2$ in BALB/c mice was $1.2 \times 10^{7} \mathrm{CFU}$. Another twenty mice in each group were challenged orally with $4.8 \times 10^{4} \mathrm{CFU}$ of $\mathrm{C} 78-3$ in $20 \mu \mathrm{l}$ PBS. The $\mathrm{LD}_{50}$ of $\mathrm{C} 78-3$ in 3-week-old $\mathrm{BALB} / \mathrm{c}$ mice was $9.5 \times 10^{2} \mathrm{CFU}$. Challenged mice were monitored for death daily for 15 days $[9,19,21,25]$.

\section{Statistical analysis}

Statistical analyses on ELISA were presented as the geometric means and standard deviations for all assays. A Mann-Whitney U Test (GraphPad Software, Inc.) was applied to contrasting the distribution of the $S$. Choleraesuis in tissues of mice. The Kaplan-Meier method (SPSS Software) was used for obtain the survival fractions following i.p. challenge of immunized mice. A $P$ value of 0.05 was considered statistically significant.

\section{Abbreviations}

S.Choleraesuis: Salmonella enterica serotype Choleraesuis; S.

suis: Streptococcus suis; Sao: Surface-anchored protein; SS2: S.suis serotype 2; RDAS: Regulated delayed attenuated strategies; LB: Luria broth; LD 50 : 50\% lethal dose; APC: Antigen-Presenting Cells; MHCl: Major Histocompatibility Complexl; Th1: T helper type 1; PBS: Phosphate buffer saline;

LPS: Lipopolysaccharide; SDS: Sodium dodecyl sulfate-polyacrylamide 


\section{Acknowledgements}

The authors thank to Roy Curtiss, III for kindly providing X7213, pRE112, pYA3493, and the antibody of Fur.

\section{Authors' contributions}

Design of the study: YA-L, HY-S and SF-W. Performed experiments: YA-L, YZD, WW-G, J-F, YY-C. Wrote the manuscript: YA-L, DF-C, XB-W, MB, SF-W. All authors have read and approved the manuscript.

\section{Funding}

This work was supported by the National Natural Science Foundation of China (31672516, 31172300, 30670079), supported by the Grant No. BE2016343 from Jiangsu province and the Priority Academic Program Development of Jiangsu Higher Education Institutions, supported by State Key Laboratory of Genetically Engineered Veterinary Vaccines (No.AGVSKLZD/ZY-201807). and a project funded by the Priority Academic Program Development of Jiangsu Higher Education Institutions (PAPD). The funding bodies have not been involved in the design of the study and collection, analysis, and interpretation of data and in writing the manuscript.

\section{Availability of data and materials}

All data generated or analysed during this study will be available from the corresponding author on reasonable request.

\section{Ethics approval and consent to participate}

Procedures involving the care and use of animals were approved by the Jiangsu Administrative Committee for Laboratory Animals (permission number SYXK-SU-2007-0005) and complied with the Jiangsu Laboratory Animal Welfare and Ethics guidelines of the Jiangsu Administrative Committee of Laboratory Animals.

\section{Consent for publication}

Not applicable.

\section{Competing interests}

None of the authors of this manuscript have any competing or financial interests.

\section{Author details \\ ${ }^{1}$ College of Veterinary Medicine, Yangzhou University, Yangzhou 225009, Jiangsu, People's Republic of China. ${ }^{2}$ Jiangsu Co-innovation Center for the Prevention and Control of Important Animal Infectious Diseases and Zoonoses, Yangzhou 225009, China. ${ }^{3}$ Key Laboratory of Animal Infectious Diseases, Ministry of Agriculture, Yangzhou University, Yangzhou, China. ${ }^{4}$ Jiangsu Key Laboratory of Preventive Veterinary Medicine, Yangzhou University, Yangzhou, China. ${ }^{5}$ Yebio Bioengineering Co., Ltd of Qingdao, Qingdao 266114, China. ${ }^{6}$ Yangzhou Uni-Bio Pharmaceutical Co., Ltd, Yangzhou 225000, Jiangsu, China. ${ }^{7}$ Department of Infectious Diseases and Immunology, College of Veterinary Medicine, University of Florida, Gainesville, FL 32611-0880, USA.}

\section{Received: 14 June 2019 Accepted: 22 April 2020} Published online: 07 May 2020

\section{References}

1. Seele J, Hillermann LM, Beineke A, et al. The immunoglobulin M-degrading enzyme of Streptococcus suis, IdeS suis, is a highly protective antigen against serotype 2. Vaccine. 2015;33(19):2207-12.

2. Roy D, Fittipaldi N, Dumesnil A, et al. The protective protein Sao (surface antigen one) is not a critical virulence factor for Streptococcus suis serotype 2[J]. Microbial Pathogenesis, 2014;67-68:31-35.

3. Li Y, Martinez G, Gottschalk M, et al. Identification of a surface protein of Streptococcus suis and evaluation of its immunogenic and protective capacity in pigs. Infect Immun. 2006;74(1):305-12.

4. Li Y, Gottschalk M, Esgleas M, et al. Immunization with recombinant Sao protein confers protection against Streptococcus suis infection. Clin Vaccine Immunol. 2007:14(8):937-43.

5. Li YA, Ji Z, Wang $X$, et al. Salmonella enterica serovar Choleraesuis vector delivering SaoA antigen confers protection against Streptococcus suis serotypes 2 and 7 in mice and pigs. Vet Res. 2017;48(1):89.
6. Curtiss R, Wanda SY, Gunn BM, et al. Salmonella enterica Serovar Typhimurium strains with regulated delayed attenuation in vivo. Infect Immun. 2009;77(3):1071-82.

7. Collins LV, Attridge S, Hackett J. Mutations at rfc or pmi attenuate Salmonella typhimurium virulence for mice. Infect Immun. 1991;59(3):1079-85.

8. Li Y, Wang S, Scarpellini G, et al. Evaluation of new generation Salmonella enterica serovar Typhimurium vaccines with regulated delayed attenuation to induce immune responses against PspA. Proc Natl Acad Sci. 2009;106(2): 593-8.

9. Ji Z, Shang J, Li Y, et al. Live attenuated Salmonella enterica serovar Choleraesuis vaccine vector displaying regulated delayed attenuation and regulated delayed antigen synthesis to confer protection against Streptococcus suis in mice. Vaccine. 2015:33(38):4858-67.

10. Riesenbergwilmes MR, Bearson B, Foster JW, et al. Role of the acid tolerance response in virulence of Salmonella typhimurium. Infect Immun. 1996:64(4): 1085-92.

11. Tsolis RM, Bäumler AJ, Stojiljkovic I, et al. Fur regulon of Salmonella typhimurium: identification of new iron- regulated genes. J Bacteriol. 1995; 177(16):4628-37.

12. Troxell B, Hassan HM. Transcriptional regulation by ferric uptake regulator (Fur) in pathogenic bacteria. Front Cell Infect Microbiol. 2013;3:59.

13. Choi $\mathrm{E}, \mathrm{Kim} \mathrm{H}$, Lee $\mathrm{H}$, et al. The Iron-sensing Fur regulator controls expression timing and levels of Salmonella Pathogenicity Island 2 genes in the course of environmental acidification. Infect Immun. 2014;82(6):2203-10.

14. Galán JE, Nakayama K, Curtiss R. Cloning and characterization of the asd gene of Salmonella typhimurium: use in stable maintenance of recombinant plasmids in Salmonella vaccine strains. Gene (Amsterdam). 1990:94(1):29-35.

15. Nakayama K, Kelly SM, Curtiss R. Construction of an ASD+ expressioncloning vector: stable maintenance and high level expression of cloned genes in a Salmonella vaccine strain. Nat Biotechnol. 1988;6(6):693-7.

16. Zhao Z, Xue Y, Wu B, Tang X, Hu R, Xu Y, Guo A, Chen H. Subcutaneous vaccination with attenuated Salmonella enterica serovar Choleraesuis C500 expressing recombinant filamentous hemagglutinin and pertactin antigens protects mice against fatal infections with both S. enterica serovar Choleraesuis and Bordetella bronchiseptica. Infect Immun. 2008;76:2157-63.

17. Zhang X, Kelly SM, Bollen WS, et al. Characterization and immunogenicity of Salmonella typhimurium SL1344 and UK-1 $\Delta c r p$ and $\Delta c d t$ deletion mutants. Infect Immun. 1997:65(12):5381.

18. Higginson EE, Ramachandran G, Panda A, Shipley ST, Kriel EH, DeTolla LJ, Lipsky M, Perkins DJ, Salerno-Goncalves R, Sztein MB, Pasetti MF, Levine MM, Tennant SM. Improved Tolerability of a Salmonella enterica Serovar Typhimurium Live-Attenuated Vaccine Strain Achieved by Balancing Inflammatory Potential with Immunogenicity. Infect Immun. 2018;86(12): e00440-18.

19. Gunn BM, Wanda SY, Burshell D, et al. Construction of recombinant attenuated Salmonella enterica Serovar Typhimurium vaccine vector strains for safety in newborn and infant mice. Clin Vaccine Immunol. 2010;17(3): 354-62.

20. Li Y, Wang S, Xin W, et al. A sopB deletion mutation enhances the immunogenicity and protective efficacy of a heterologous antigen delivered by live attenuated Salmonella enterica vaccines. Infect Immun. 2008;76(11):5238-46

21. Matulova M, Havlickova H, Sisak F, et al. SPI1 defective mutants of Salmonella enterica induce cross-protective immunity in chickens against challenge with serovars Typhimurium and Enteritidis. Vaccine. 2013:31(31): 3156-62.

22. Foster JW, Hall HK. Effect of Salmonella typhimurium ferric uptake regulator (fur) mutations on iron- and $\mathrm{pH}$-regulated protein synthesis. J Bacteriol. 1992;174(13):4317-23.

23. Huoying S, Javier S, Brenneman KE, et al. Live recombinant Salmonella Typhi vaccines constructed to investigate the role of rpos in eliciting immunity to a heterologous antigen. PLoS One. 2010;5(6):e11142.

24. Galen JE, Curtiss R. The delicate balance in genetically engineering live vaccines. Vaccine. 2014:32(35):4376-85.

25. Kong Q, Liu Q, Jansen AM, Curtiss R. Regulated delayed expression of rfc enhances the immunogenicity and protective efficacy of a heterologous antigen delivered by live attenuated Salmonella enterica vaccines. Vaccine. 2010:28(37):6094-103

26. Zhu $L$, Zhao X, Yin Q, et al. Mucosal IgA and IFN- $\gamma+$ CD8 T cell immunity are important in the efficacy of live Salmonella enteria serovar Choleraesuis vaccines. Sci Rep. 2017;7:46408. 
27. Xinxin Z, Sheng L, Qinlong D, et al. Regulated delayed attenuation enhances the immunogenicity and protection provided by recombinant, Salmonella, enterica, serovar Typhimurium vaccines expressing serovar Choleraesuis O-polysaccharides. Vaccine. 2018:36(33):5010-9.

28. Tsai C. A sensitive silver stain for detecting lipopolysaccharides in polyacrylamide gels. Anal Biochem. 1982;119(1):115-9.

29. Witholt B, Boekhout M, Brock M, et al. An efficient and reproducible procedure for the formation of spheroplasts from variously grown Escherichia coli. Anal Biochem. 1976;74(1):160-70.

30. Kang HY, Dozois CM, Tinge SA, et al. Transduction-mediated transfer of unmarked deletion and point mutations through use of Counterselectable suicide vectors []]. J Bacteriol. 2002;184(1):307-12.

31. Wang S, Li Y, Scarpellini G, et al. Salmonella vaccine vectors displaying delayed antigen synthesis in vivo to enhance immunogenicity. Infect Immun. 2010;78(9):3969-80.

32. Hitchcock P. Morphological heterogeneity among Salmonella lipopolysaccharide chemotypes in silver-stained polyacrylamide gels. J Bacteriol. 1983;154:269-77.

33. Kang HY. Immune responses to recombinant pneumococcal PspA antigen delivered by live attenuated Salmonella enterica Serovar Typhimurium vaccine. Infect Immun. 2002;70(4):1739-49.

34. Schindelin J, Rueden CT, Hiner MC, et al. The ImageJ ecosystem: an open platform for biomedical image analysis. Mol Reprod Dev. 2015;82(7-8):518-29.

35. Rd CS. Chromosomal Aberrations associated with mutations to bacteriophage resistance in Escherichia Coli. J Bacteriol. 1965;89(1):28.

36. Pashine A, John B, Rath S, George A, Bal V. Th1 dominance in the immune response to live Salmonella typhimurium requires bacterial invasiveness but not persistence. Int Immunol. 1999 Apr;11(4):481-9.

\section{Publisher's Note}

Springer Nature remains neutral with regard to jurisdictional claims in published maps and institutional affiliations.

Ready to submit your research? Choose BMC and benefit from:

- fast, convenient online submission

- thorough peer review by experienced researchers in your field

- rapid publication on acceptance

- support for research data, including large and complex data types

- gold Open Access which fosters wider collaboration and increased citations

- maximum visibility for your research: over $100 \mathrm{M}$ website views per year

At $\mathrm{BMC}$, research is always in progress.

Learn more biomedcentral.com/submissions 